\title{
Habilidades Sociais como Preditoras do Envolvimento com Álcool e Outras Drogas: Um estudo exploratório
}

\author{
Lucas Guimarães Cardoso de Sá * \\ Universidade Federal do Maranhão, São Luís, Brasil \\ Zilda Aparecida Pereira Del Prette \\ Universidade Federal de São Carlos, São Carlos, Brasil
}

\begin{abstract}
RESUMO
O objetivo deste estudo foi investigar relações entre habilidades sociais e envolvimento com álcool, crack, maconha e nicotina em uma amostra composta por 47 pessoas admitidas em tratamento ambulatorial por abuso ou dependência dessas drogas. Foram utilizados o Inventário de Habilidades Sociais e o Teste de Triagem do Envolvimento com Álcool, Cigarro e Outras Substâncias. Os resultados mostraram correlações significativas entre as variáveis, com a variância do envolvimento com substâncias sendo explicada entre $10 \%$ e $44 \%$ pelas habilidades sociais. Os resultados apoiam a hipótese de que déficits no repertório geral de habilidades sociais não são necessariamente uma característica do abuso ou dependência de álcool e outras drogas, mas que classes e contextos específicos podem predizer o envolvimento com as substâncias.
\end{abstract}

Palavras-chave: habilidades sociais; álcool; cocaína crack; drogas.

\section{ABSTRACT \\ Social Skills as Predictors of Alcohol and Other Drugs Involvement: An Exploratory Study}

The aim of this study was to investigate the relations between social skills and involvement with alcohol, crack cocaine, marijuana and nicotine in a sample composed by 47 people admitted to outpatient treatment for abuse or dependence on these drugs. Social Skills Inventory and Alcohol, Smoking and Substance Involvement Screening Test were used. The results showed significant correlations between variables, with the substances involvement variance being explained between $10 \%$ and $44 \%$ by social skills. The results support the hypothesis that deficits in the general repertoire of social skills are not necessarily a characteristic of abuse or dependence on alcohol and other drugs, but specific classes and contexts can predict the involvement with substances.

Keywords: social skills; alcohol; crack cocaine; drugs.

A Dependência de Substâncias pode ser caracterizada por um conjunto de sintomas cognitivos, comportamentais e fisiológicos indicando constante autoadministração de substâncias psicoativas, ainda que isso traga problemas significativos ao indivíduo e resulte em perda do controle voluntário do consumo, tolerância aos efeitos da droga e a sintomas específicos quando é realizada abstinência (American Psychiatric Association [APA], 2002). Os comportamentos dependentes fazem parte de um transtorno complexo, no qual estão envolvidos fatores genéticos, fisiológi- cos, sociais, culturais e psicológicos que interagem entre si e atingem as pessoas de várias maneiras, por diferentes motivos e em contextos diversos (Donovan, 2009).

Em relação ao fator psicológico, uma das explicações aceitas é que o comportamento dependente seria mantido por uma maneira desadaptativa de lidar com situações cotidianas de estresse, sejam em família, no trabalho, com amigos ou mesmo em breves relacionamentos com desconhecidos (Monti, Kadden, Rohsenow, Cooney, \& Abrams, 2005). O estresse, 
nesse caso entendido como um desequilíbrio entre as exigências do ambiente e os recursos do indivíduo, poderia levar aqueles que não têm um nível satisfatório desses recursos a buscar a droga como encorajadora para enfrentar situações difíceis ou como alívio do mal-estar causado por tais situações (Forys, McKellar, \& Moos, 2007; Monti et al., 2005). Brown et al. (1990), em estudo com 129 pacientes, já haviam relatado que estressores anteriores ao tratamento estavam direta ou indiretamente relacionados ao uso de álcool e que estresse severo após tratamento aumenta o risco de uma recaída, ou seja, volta ao consumo compulsivo da droga após período de abstinência.

Para diminuir a probabilidade de voltar a beber ou usar outras drogas é preciso auxiliar a pessoa a discriminar quais situações trazem risco de usar a substância e ensinar-lhe novas formas de enfrentar adequadamente tais momentos adversos, aumentando a sua autoeficácia nesse enfrentamento (Marlatt \& Witkiewitz, 2009). De acordo com Rangé e Marlatt (2008), é importante mostrar como resistir à droga, fazendo com que a pessoa se envolva e treine os comportamentos ensinados, participando do seu próprio processo de mudança. Esse modelo, conhecido como Prevenção da Recaída, está descrito com detalhes em Marlatt e Donovan (2009).

Diversos autores listaram diferentes habilidades, interpessoais e intrapessoais, necessárias durante o processo de prevenção da recaída (Araújo, Oliveira, Pedroso, \& Castro, 2009; Bordin, Zanelatto, Figlie \& Laranjeira, 2010; Ciribelli, Luiz, Gorayeb, Domingos, \& Filho, 2008; Monti et al., 2005, Murta \& Tróccoli, 2005; Rangé \& Marlatt, 2008; Rohsenow, Martin \& Monti, 2005; Sakiyama, Ribeiro \& Padin, 2012). Nesse contexto, o repertório de habilidades sociais é considerado uma importante variável no processo de prevenção da recaída. Esse construto é descritivo e representa as classes de comportamentos sociais que a pessoa dispõe para completar de forma bem sucedida uma determinada tarefa social (Del Prette \& Del Prette, 2010). Elas são condição necessária, ainda que não suficiente, para a competência social, definida como a "capacidade de articular pensamentos, sentimentos e ações em função de objetivos pessoais e de demandas da situação e da cultura, gerando consequências positivas para o indivíduo e para a sua relação com as demais pessoas" (Del Prette \& Del Prette, 2005, p. 33).
A suposição, portanto, é que o dependente de substâncias psicoativas necessita de um bom repertório de habilidades sociais para um efetivo enfrentamento de situações estressoras e de risco. Um repertório deficitário poderia levar a um conjunto de situações e sentimentos negativos que dificultariam as tentativas de manutenção da abstinência (Ferreira \& Bernik, 2001; Sakiyama et al., 2012; Villalba, 1995). Rangé e Marlatt (2008, p.92) destacam que enfrentamento efetivo requer "habilidades sociais básicas, comportamentos assertivos e habilidades de confronto, que incluem a capacidade de identificar situações de risco, lidar com emoções e fazer reestruturações cognitivas".

No processo de manutenção da abstinência, um bom repertório de habilidades sociais contribuiria para evitar que uma situação social, inicialmente neutra, se tornasse negativa ou conflituosa e pudesse representar um gatilho para o uso. Também aumentaria a probabilidade de consecução de metas (por exemplo, identificar e afastar-se das situações de risco), manutenção do autocontrole, melhora da autoestima e da qualidade das relações com as demais pessoas (Villalba, 1995). Assim, além de produzir comportamentos mais adaptativos diante de estressores ou humores negativos gerados nos relacionamentos sociais do dia a dia, proporcionaria relações interpessoais satisfatórias para o indivíduo, protegendo-o de uma recaída.

Desde a década de 1970, há estudos apontando que um bom repertório de habilidades sociais está de alguma forma ligado à manutenção da abstinência de álcool ou outras drogas (ver Felicíssimo, Casela, \& Ronzani, 2013). Como exemplos, Van Hasselt, Hersen e Milliones (1978) fizeram uma revisão sobre os diversos estudos que até então tinham empregado o treinamento de habilidades sociais como técnica de intervenção, sugerindo sua utilidade para o tratamento de dependentes de álcool e outras drogas. Oei e Jackson (1982) mostraram uma forte relação entre aumento do repertório de habilidades sociais e decréscimo no consumo de álcool. Monti et al. (1990) verificaram que, nos meses seguintes a um treinamento de habilidades de comunicação em um programa de tratamento para o alcoolismo, houve uma queda significativa na taxa de uso de álcool, quando comparada àquela registrada em um tratamento no qual não houve o mesmo treinamento. Em outro estudo, Monti, Rohsenow, Michalec, Martin e Abrams (1997) ensinaram a paci- 
entes usuários de cocaína, habilidades para lidar com situações de frustração, raiva, sentimentos negativos e pressão social para usar a droga, entre outras. Os resultados mostraram que, nos três meses seguintes, esses pacientes fizeram menor uso da droga e tiveram períodos mais curtos de recaída quando comparados àqueles de um grupo controle. Em sentido inverso, Scheier, Botvin, Diaz e Griffin (1999) encontraram que medidas de baixa competência social e pouca habilidade de recusa estavam relacionadas a consumo de álcool.

Nos últimos anos, pesquisas seguem indicando que a abstinência de drogas parece estar relacionada ao exercício bem sucedido de componentes das habilidades sociais, muitas vezes após o indivíduo passar por um treinamento delas, melhorando seu desempenho (Ball et al., 2007; Budney, Roffman, Stephens, \& Walker, 2007; Hasking \& Oei, 2007; Litt, Kadden, Kabela-Cormier, \& Petry, 2008, Mattoo, Chakrabarti, \& Anjaiah, 2009, Mares \& Torres, 2010; Marijuana Treatment Project Research Group, 2004; Witkiewitz, Villarroel, Hartzler, \& Donovan, 2011). Outras, ainda mais focadas, identificaram habilidades específicas significativamente ligadas ao consumo e abstinência de álcool e outras drogas: para Barkin, Smith e DuRant (2002) e Wagner e Oliveira (2007), recusar oferta de drogas; para Cunha, Carvalho, Kolling, Silva e Kristensen (2007), conversação, manejo de situações de conflito e expressão de sentimentos positivos; para Wagner e Oliveira (2009), autoexposição a desconhecidos, situações novas e de controle da agressividade; para Cleveland e Harris (2010), evitação de situações de risco e resolução de problemas.

Apesar de diversas investigações apontarem a ligação entre habilidades sociais e abstinência de substâncias psicoativas, evidências empíricas quanto a características dessa relação ainda não se apresentam suficientemente sustentáveis. Por exemplo, embora estudos estrangeiros tenham apontado algumas classes de habilidades sociais ligadas à abstinência de álcool e outras drogas, não se sabe com precisão a força dessa relação e, menos ainda, o quanto o repertório dessas habilidades pode interferir no envolvimento com álcool e outras drogas. No Brasil, até o momento, a maior parte dos estudos comparou o repertório de habilidades sociais de dependentes químicos com o de não dependentes ou o repertório de um mesmo dependente, antes e depois de uma intervenção (ver Aliane,
Lourenço, \& Ronzani, 2006; Wagner \& Oliveira, 2009, Wagner, Silva, Zanettelo, \& Oliveira, 2010).

Em geral, afirma-se que um bom repertório de habilidades sociais está relacionado à abstinência e um repertório menos elaborado à dependência de álcool e outras drogas. Contudo, não parece ter sido especificado ainda se isto é válido para o repertório geral, para repertórios específicos, para ambos ou sequer se a afirmação é inteiramente válida. Também não há dados mostrando se o envolvimento com diferentes tipos de drogas está relacionado a diferentes habilidades, o que implicaria a necessidade de intervenções distintas, conforme o tipo de dependência. Por exemplo, o processo de abstinência de crack poderia requerer uma habilidade que no de álcool teria menor importância ou menor efeito. Por todas as lacunas existentes, novos estudos são de grande importância para o maior conhecimento da área. Desta maneira, o objetivo deste estudo foi investigar possíveis relações entre habilidades sociais e envolvimento com substâncias, mais especificamente o quanto o repertório dessas habilidades pode predizer o nível de envolvimento com álcool, crack, maconha e nicotina.

\section{MÉTODO}

\section{Participantes}

Foram contatadas 54 pessoas, mas sete foram excluídas por não atenderem a ao menos um dos critérios de inclusão previamente estabelecidos: (a) ter mais de 18 anos de idade, (b) não estar sob efeito de álcool ou outra substância no momento da aplicação e (c) não possuir comorbidade aparente, que incapacitasse a resposta aos instrumentos. Portanto, a amostra foi composta por 47 pessoas admitidas em tratamento ambulatorial por abuso ou dependência de álcool ou outras drogas em um Centro de Atenção PsicossocialÁlcool e Drogas (CAPS-AD). A idade variou entre 21 e 74 anos $(M=34.95 ; D P=13.54), 89,3 \%$ eram homens, $44.7 \%$ tinham no máximo primeiro grau completo, $38.3 \%$ no máximo segundo grau completo e $17 \%$ ao menos iniciaram curso superior. Do total, 46 pessoas possuíam algum nível de envolvimento com álcool, 22 com crack, 24 com maconha e 39 com nicotina. Como é comum no tratamento da dependência química, os pacientes possuíam envolvimento com múltiplas drogas simultaneamente. O envolvimento exclusivo com álcool foi relatado por 18 pessoas e 
com o crack apenas uma. Outros 17 apresentaram envolvimento com álcool, crack e maconha simultaneamente, sete com álcool e maconha e quatro com álcool e crack.

\section{Instrumentos}

Foram utilizados o Inventário de Habilidades Sociais (IHS-Del Prette), de Del Prette e Del Prette (2001) e o Alcohol, Smoking and Substance Involvement Screening Test (ASSIST), na versão em português 'Teste de Triagem do Envolvimento com Álcool, Cigarro e Outras Substâncias', com indícios de validade apresentados para o contexto brasileiro por Henrique, Micheli, Lacerda, Lacerda e Formigoni (2004).

O IHS-Del Prette é um instrumento de autorrelato para avaliação de habilidades sociais, com consistência interna adequada (alfa de Cronbach de 0.75) e indicadores de validade e fidedignidade amplamente atestados em diversos estudos ${ }^{1}$. Contém 38 itens, cada um descrevendo uma situação de relação interpessoal e uma demanda de habilidade para reagir àquela situação. O respondente deve estimar a frequência com que reage da forma sugerida em cada item, considerando o total de vezes que se encontrou na situação descrita, e estimar a frequência de sua resposta em escala tipo Likert, com cinco pontos, variando de zero (nunca ou raramente) a quatro (sempre ou quase sempre). O IHS-Del-Prette produz um escore geral, referenciado à norma em termos de percentis, e escores em cinco subescalas de habilidades sociais: F1 (Enfrentamento e Autoafirmação com Risco), F2 (Autoafirmação na Expressão de Sentimento Positivo), F3 (Conversação e Desenvoltura Social), F4 (Autoexposição a Desconhecidos e Situações Novas) e F5 (Autocontrole da Agressividade).

O ASSIST, instrumento desenvolvido sob coordenação da Organização Mundial de Saúde, possui oito questões que investigam o uso de nove diferentes tipos de drogas nos três meses anteriores a aplicação (neste estudo foram avaliados apenas o envolvimento com bebidas alcoólicas, crack/cocaína, maconha e nicotina). O escore que mede o nível de envolvimento com as diversas substâncias pode variar de 0 a 39. Quanto maior o valor, maior o envolvimento com o tipo de droga investigada. A consistência interna do instrumento, avaliada pelo coeficiente alfa de Cronbach, foi considerada boa, sendo 0.80 para álcool, 0.80 para tabaco, 0.79 para maconha e 0.81 para cocaína.

\section{Procedimentos}

A aplicação dos instrumentos foi individual, ocorreu no processo de avaliação inicial do paciente e sempre no seu local de tratamento. O pesquisador fazia a pergunta e solicitava a resposta, anotando-a no formulário do respondente. O ASSIST foi aplicado primeiro e logo em seguida o IHS-Del Prette. As respostas foram transformadas em códigos numéricos da escala de frequência e digitadas em uma planilha da versão 18 do programa de computador PASW Statistics. A análise de distribuição em histogramas sugeriu distribuição normal, corroborada por meio do teste Kolmogorov-Smirnov. Desta maneira, os dados foram analisados estatisticamente, por meio de correlação de Pearson e regressão linear simples. Os escores do ASSIST para álcool, crack, maconha e nicotina foram correlacionados um a um com cada uma das 38 variáveis do IHS-Del Prette, além do seu escore geral e dos cinco escores fatoriais. O manual do IHS-Del Prette permite a análise considerando escore total, escores fatoriais e valores de cada item. Essa posição é coerente com a base conceitual do campo das habilidades sociais que as caracterizam como situacionais (Del Prette \& Del Prette, 2001; 2005).

As correlações indicadas como significativas foram submetidas à análise de regressão linear simples, método Enter. O envolvimento com a substância foi utilizado como variável critério e o resultado do IHSDel Prette como variável preditora. Desta maneira, foi testado o quanto o envolvimento com cada uma das substâncias poderia ser explicado pelo repertório de habilidades sociais dos indivíduos.

\section{RESULTADOS}

O envolvimento médio dos participantes com álcool foi de 26.59 pontos na escala do ASSIST ( $D P=$ $10.48)$, com crack de $31.64(D P=8.17)$, maconha de $25.21(D P=10.80)$ e nicotina de $23.92(D P=7.71)$. De acordo com as instruções do ASSIST, escores acima de 26 são indicativos de necessidade de tratamento intensivo. Os resultados elevados já eram esperados, uma vez que a amostra foi composta por pessoas admitidas para tratamento do abuso ou dependência de substâncias.

Não foram encontradas diferenças significativas que indicassem maior número de participantes com repertório deficitário em habilidades sociais, se com- 
parados àqueles com repertório bom ou elaborado. Pelo contrário, para os fatores um, dois e quatro, houve um número significativamente maior de participantes sem déficits que com déficit (Tabela 1). Para esta análise foram utilizados os dados normativos descritos no manual de aplicação, apuração e interpretação do IHS-Del Prette (Del Prette \& Del Prette, 2001).

Tabela 1

Número de Participantes Com Repertório Deficitário e Com Repertório Bom ou Elaborado No Escore Total e Fatores Do IHS-Del Prette

\begin{tabular}{cccc}
\hline Habilidades sociais & Repertório deficitário & $\begin{array}{c}\text { Repertório bom } \\
\text { ou elaborado }\end{array}$ & $\mathbf{X}^{2}$ \\
\hline Escore Total & 20 & 27 & 1,04 \\
F1-Enfrentamento e Autoafirmação com Risco & 13 & 34 & $9.38^{* *}$ \\
F2-Autoafirmação na Expressão de Sentimento Positivo & 9 & 38 & $17.89^{* * *}$ \\
F3-Conversação e Desenvoltura Social & 19 & 28 & 1.72 \\
F4-Autoexposição a Desconhecidos e Situações Novas & 13 & 34 & $9.38^{* *}$ \\
F5-Autocontrole da Agressividade & 26 & 21 & 0.53 \\
\hline
\end{tabular}

Nota. $* *$ valores muito significativos $\mathrm{p}<.01 * * *$ valores altamente significativos $\mathrm{p}<.001$

Como se observa na Tabela 2, oito classes de habilidades sociais foram significativamente correlacionadas ao envolvimento com álcool, quatro ao envolvimento com crack, duas ao envolvimento com nicotina e nenhuma ao envolvimento com maconha. Destaca-se que todas as correlações significativas entre habilidades sociais e envolvimento com álcool são negativas, ou seja, quanto maior o envolvimento com álcool, menor o repertório de habilidades sociais em geral e em outras específicas. Para o envolvimento com crack, também houve correlação negativa quanto a lidar com críticas, mas destacam-se duas correlações positivas: quanto maior o envolvimento com crack, maiores as habilidades para discordar de autoridade e encerrar conversação. No caso da nicotina, quanto maior o envolvimento com a substância, menores as habilidades para reagir a elogios e para lidar com críticas dos pais.

Tabela 2

Correlações Significativas Entre Habilidades Sociais e Envolvimento Com Álcool, Crack, Nicotina e Maconha

\begin{tabular}{lcc}
\hline Envolvimento com drogas & Habilidades sociais & $\begin{array}{c}\text { Coeficiente de correlação de } \\
\text { Pearson }(r)\end{array}$ \\
\hline & Escore total de habilidades sociais & $-0.35^{*}$ \\
F1 - Enfrentamento e autoafirmação com risco & $-0.31^{*}$ \\
F2 - Conversação e desenvoltura social & $-0.39^{* *}$ \\
& F3 - Autoexposição a desconhecidos e situações novas & $-0.33^{*}$ \\
& Falar a público desconhecido & $-0.36^{*}$ \\
Envolvimento com crack & Abordar para relacionamento sexual & $-0.32^{*}$ \\
& Reagir a elogio & $-0.37^{*}$ \\
\hline Envolvimento com nicotina & Abordar autoridade & $-0.48^{* * *}$ \\
\hline Envolvimento com maconha & Discordar de autoridade & $0.68^{* *}$ \\
\hline
\end{tabular}

Nota. *valores significativos $p<.05^{* *}$ valores muito significativos $p<.01{ }^{* * *}$ valores altamente significativos $p<.001$ 
Como foram encontradas correlações significativas entre algumas das variáveis investigadas, foi testado o quanto o envolvimento com álcool, crack ou nicotina pode ser explicado por habilidades sociais específicas. Os resultados da regressão linear indicaram que quatro das 38 habilidades avaliadas por meio do IHS-Del Prette, além do escore geral e dos escores fatoriais um, três e quatro, são preditores significativos do envolvimento com álcool. As habilidades sociais explicam entre $8 \%$ e $21 \%$ da variância do envolvimento com álcool, sendo que para cada ponto de aumento do repertório em habilidades sociais, medido pelo IHSDel Prette, o envolvimento com álcool diminui entre 0.25 e 3.28 pontos, medido pelo ASSIST (Tabela 3 ).

Tabela 3

Resumo das Análises de Regressão Linear para Habilidades Sociais como Preditoras do Envolvimento Com Álcool e Outras Drogas

\begin{tabular}{cccccc}
\hline Variável explicativa & Variável de critério & $\mathbf{R}^{\mathbf{2}}$ & $\mathbf{a}$ & $\mathbf{b}$ & $\boldsymbol{t}$ \\
\hline Falar a público desconhecido & & 0.11 & 31.34 & -2.33 & $-2.55^{*}$ \\
Abordar para relacionamento sexual & & 0.09 & 31.53 & -2.50 & $-2.27^{*}$ \\
Reagir a elogio & & 0.11 & 33.41 & -3.08 & $-2.61^{* *}$ \\
Abordar autoridade & Envolvimento com álcool & 0.21 & 33.22 & -3.28 & $-3.61^{* * *}$ \\
HS geral & & 0.10 & 45.95 & -0.25 & $-2.47^{*}$ \\
Enfrentamento e Autoafirmação com Risco & & 0.08 & 38.49 & -1.19 & $-2.16^{*}$ \\
Conversação e Desenvoltura Social & & 0.13 & 40.28 & -2.17 & $-2.82^{* *}$ \\
Autoexposição a Desconhecidos e Situações Novas & & 0.09 & 34.77 & -2.40 & $-2.33^{*}$ \\
\hline Discordar de autoridade & & 0.44 & 21.25 & 4.31 & $4.20^{* * *}$ \\
Encerrar conversação & Envolvimento com crack & 0.30 & 24.16 & 3.50 & $3.19^{* *}$ \\
Lidar com críticas dos pais & & 0.31 & 38.05 & -4.03 & $-3.21^{* *}$ \\
Lidar com críticas justas & & 0.17 & 37.71 & -2.67 & $-2.27^{*}$ \\
\hline Reagir a elogio & \multirow{2}{*}{ Envolvimento com nicotina } & 0.10 & 28.21 & -2.11 & $-2.25^{*}$ \\
Lidar com críticas dos pais & & 0.10 & 28.06 & -2.09 & $-2.31^{*}$ \\
\hline
\end{tabular}

Nota. *valores significativos $\mathrm{p}<.05 * *$ valores muito significativos $\mathrm{p}<.01 * * *$ valores altamente significativos $\mathrm{p}<.001$

Na Tabela 3 também é possível observar que quatro variáveis do IHS-Del-Prette foram preditoras do envolvimento com crack. Lidar com Críticas dos Pais e $\mathrm{Li}$ dar com Críticas Justas explicaram $31 \%$ e $17 \%$ do envolvimento com a droga, e para cada ponto de aumento nessas habilidades houve decréscimo de $4.03 \mathrm{e}$ 2.67 pontos no envolvimento com crack. Por outro lado, Discordar de Autoridade e Encerrar Conversação explicaram respectivamente $44 \%$ e $30 \%$ do envolvimento com a droga, sendo que cada ponto de acréscimo nestas habilidades ocasionou um aumento de 4.31 e 3.50 pontos no envolvimento com a substância.

Por fim, duas variáveis do IHS-Del Prette foram preditoras do envolvimento com nicotina. Reagir a Elogios e Lidar com Críticas dos Pais explicaram, cada uma, $10 \%$ do envolvimento com a substância, sendo que cada aumento no repertório dessas habilidades veio acompanhado por um decréscimo de $2.11 \mathrm{e}$ 2.09 pontos no envolvimento com nicotina.

\section{DISCUSSÃO}

Os resultados deste estudo podem ser resumidos em duas linhas principais de discussão: (1) ausência de evidências para confirmar a hipótese de que um bom repertório de habilidades sociais está relacionado à abstinência e um repertório deficitário à dependência de álcool e outras drogas e (2) evidências de apoio à hipótese de que há relação entre habilidades sociais e envolvimento com álcool e outras drogas. As hipóteses, embora distintas, são muitas vezes tratadas como conflitantes quando, de fato, não são. A ausência de déficits, por exemplo, não implica necessariamente em ausência de relação entre as variáveis.

Para a possível refutação da hipótese de que déficits no repertório de habilidades sociais são comuns em dependentes de álcool e outras drogas, como tradicionalmente descrito na literatura, os dados deste estudo mostraram que as diferenças significativas, 
quando ocorreram, indicaram maior número de participantes com bom repertório que com repertório deficitário. Ou seja, o contrário do que afirma a referida hipótese. Embora existam dependentes químicos com repertório deficitário, não é possível afirmar que isso seja significativo ou uma regra geral.

Sá e Del Prette (2012) mostram evidências nesse sentido, indicando que o repertório de habilidades sociais de dependentes químicos pode ser considerado suficientemente bom antes mesmo de uma intervenção. Cunha, Peuker e Bizarro (2012) não encontraram resultados significativos de associação entre consumo de risco de álcool e déficits em habilidades sociais em universitários. Aliane et al. (2006) e Cunha et al. (2007), também indicam ausência de evidências que sustentem tal hipótese. No primeiro caso não foram encontradas quaisquer diferenças significativas quando comparado o repertório de habilidades sociais de uma amostra de dependentes de álcool com o de uma amostra de não dependentes. No segundo, o repertório de habilidades sociais apresentado por alcoolistas não sugeria necessidade de treinamento na maior parte dos casos. Em resumo, investigações diferentes, utilizando métodos distintos, alcançaram resultados semelhantes.

E é mesmo plausível que a dependência química não seja resultado da incapacidade de realização da maioria das tarefas sociais rotineiras, sem uma relação direta com o contexto da adicção de cada indivíduo. Por exemplo, faria pouco sentido propor que dependentes de cocaína tenham mais dificuldade que não dependentes em pedir para um lojista trocar um produto defeituoso. Muitos podem ter tal dificuldade, mas diversos não dependentes também a têm, ou seja, não é uma situação que diferencia os grupos.

Essa diferenciação pode ser a chave para propor uma hipótese alternativa. A sugestão é que dependentes químicos, comparados a não dependentes, teriam repertório de habilidades sociais significativamente menor ou deficitário apenas em contextos que envolvam o consumo de substâncias ou em determinados comportamentos, fora deste contexto, mas que de alguma forma estão ligados à dependência. No primeiro caso espera-se, por exemplo, que um alcoolista tenha um repertório significativamente menor para recusar oferta de bebida que uma pessoa abstêmia. Nesse caso, o problema não seria a habilidade para recusar em si, mas recusar em um contexto diretamente ligado à dependência. No segundo, a ideia é que determinadas habilidades podem influenciar o consumo de substâncias, mesmo que indiretamente. Por exemplo, estudos como os de Wagner e Oliveira (2009) e Wagner et al. (2010) dão indícios de que autocontrole da agressividade poderia ser uma dessas habilidades. Déficits nessa classe específica poderiam dificultar a abstinência, uma vez que o uso de maconha seria a maneira mais fácil ou prática para aliviar as consequências negativas resultantes da falta de autocontrole.

Com essa proposta é possível seguir para uma segunda etapa da discussão, baseada no fato de que importantes correlações foram encontradas em habilidades bastante específicas, o que novamente parece indicar a particularidade da relação entre habilidades sociais e envolvimento com substâncias psicoativas. O primeiro resultado a ser destacado é o que indica que todas as correlações significativas entre habilidades sociais e envolvimento com álcool foram negativas. É possível supor, portanto, que maior repertório de determinadas habilidades sociais está ligado a menor envolvimento com álcool. Isto corrobora resultados relatados por Oei e Jackson (1982), que mostraram uma forte relação entre aumento do repertório de habilidades sociais e decréscimo no consumo de álcool. No Brasil, Oliveira (2010) também encontrou correlação significativa negativa entre envolvimento com álcool e habilidades de autocontrole da agressividade ou a situações aversivas. Conforme estes resultados, conclui-se que uma maneira (certamente não a única) de reduzir o envolvimento com álcool seria promover determinadas habilidades sociais especificamente associadas a esse envolvimento.

É interessante apontar que, exceção feita a "reagir a elogios", as demais habilidades com resultados significativos envolvem situações em que a pessoa deve se expor e dar início a comportamentos da classe de habilidades sociais. A bebida pode ser utilizada, portanto, como uma forma de facilitar o desempenho desses comportamentos. Se o indivíduo aprende a se comportar de maneira alternativa, mais adequada, a bebida pode se tornar menos necessária e, portanto, menos provável de ser consumida. Desta forma, considerando os resultados obtidos, para diminuir o envolvimento com álcool, seria interessante investir no 
ensino das habilidades relacionadas a enfrentamento com risco, conversação e desenvoltura social e autoexposição a desconhecidos e situações novas.

Para o envolvimento com crack, das quatro correlações significativas, duas foram negativas, como esperado, mas ao contrário do encontrado para o envolvimento com o álcool, as habilidades estiveram relacionadas a responder adequadamente a um comportamento iniciado por outra pessoa, não a iniciar o comportamento. Nesse caso, poderia ser promissor investir no ensino de habilidades como Lidar com Críticas dos Pais e Críticas Justas, uma vez que a previsão é de que com isso haja redução no envolvimento com a droga. As outras duas correlações significativas envolveram comportamentos iniciados pela própria pessoa, Discordar de Autoridade e Encerrar Conversação, mas foram surpreendentemente positivas: quanto maior o repertório de tais habilidades maior o envolvimento com o crack. Uma possibilidade é que este resultado seja característica de uma propensão à impulsividade e tomada de risco em dependentes de crack, conforme relatado por Kessler et al. (2012). Outra, que não descarta a primeira, é que tais comportamentos tenham se desenvolvido justamente no processo de estabelecimento da dependência, pois, para conseguir a droga, ilegal, é preciso iniciar diversas interações. No envolvimento com álcool, substância legal, isso não seria necessário, já que o indivíduo pode, por exemplo, comprar a bebida em um supermercado sem ter que interagir com praticamente ninguém.

Neste caso, em que não é eticamente aceitável intervir no sentido de redução do repertório dessas habilidades, uma alternativa pode ser seu redirecionamento, utilizando-as sistematicamente em conjunto com comportamentos mais efetivos e adequados. Esta relação estabelecida com novos comportamentos manteria ou até elevaria o repertório de tais habilidades, mas, por estarem ligadas a comportamentos saudáveis, a associação que possuíam anteriormente, de envolvimento com a droga, ficaria enfraquecida.

As correlações negativas significativas entre o envolvimento com nicotina e habilidades para Reagir a Elogios e Lidar com Críticas dos Pais, parecem seguir, ao menos em parte, a explicação proposta para a relação entre habilidades sociais e o envolvimento com crack. As duas são habilidades em que o indiví- duo deve responder a um comportamento iniciado por outra pessoa, de maneira que quanto melhor o repertório destas habilidades, menor o envolvimento com a nicotina.

Por fim, a ausência de correlações significativas entre habilidades sociais e o envolvimento com maconha não deixa de ser surpreendente, embora estudos anteriores já sugerissem que isto não fosse improvável, uma vez que boa parte dos resultados envolvendo tais variáveis foram não significativos (Wagner \& Oliveira, 2009, Wagner et al., 2010). Uma possível justificativa é de que, por ser uma droga perturbadora do Sistema Nervoso Central (SNC), o indivíduo buscaria seu efeito para lidar com questões mais pessoais e não para se expor ou para lidar com ser exposto, como no caso do álcool e do crack. Seria, portanto, uma droga com impacto menos social e mais intrapessoal. É importante que pesquisas futuras deem atenção a esta lacuna e investiguem o tema com mais cuidado.

As relações encontradas entre as variáveis de "envolvimento com drogas" e as de habilidades sociais ensejam algumas especulações, considerando que os dados deste estudo não permitem uma associação direta. Assim, sem dispensar cautela na interpretação, é possível pensar que o repertório de comportamentos sociais do indivíduo pode influenciar a escolha por tipos distintos de drogas. O envolvimento com álcool, uma droga depressora do SNC, parece estar relacionado principalmente a comportamentos interpessoais em que o indivíduo precisa iniciar a interação. $\mathrm{O}$ efeito de euforia inicial proporcionado por esta droga pode aumentar a frequência desses comportamentos, embora dificulte que eles sejam, de fato, aprendidos. Assim, é estabelecida uma relação negativa, em que maior envolvimento com o álcool está associado a menor repertório de habilidades sociais.

De maneira inversa, no caso do envolvimento com crack, droga estimulante do SNC, a relação com comportamentos de iniciar interação é positiva, ou seja, a droga não parece ter a função de facilitar a busca por melhor desempenho destes comportamentos. Nesse caso, a correlação negativa encontrada foi para habilidades ligadas a responder a comportamentos iniciados por outras pessoas. Aqui, a hipótese é que a estimulação proporcionada pela droga pode facilitar tais respostas. Resultados semelhantes encontrados para o envolvimento com a nicotina, uma droga também 
estimulante, parecem corroborar essa explicação. Assim, da mesma maneira que o consumo de álcool serviria para facilitar determinados comportamentos de iniciar interações, o de crack serviria para facilitar aqueles ligados a responder a interações iniciadas por outras pessoas.

Até mesmo o fato de não terem sido encontradas correlações significativas entre habilidades sociais e envolvimento com maconha, droga perturbadora do SNC, pode ser considerado parte do argumento que sustenta a hipótese de que cada tipo de droga está ligado de forma distinta a diferentes tipos de habilidades sociais. Como ressaltado anteriormente, são apenas hipóteses. É fundamental que outras pesquisas sejam realizadas com o objetivo de expandir a discussão sobre o assunto.

De qualquer forma, por tudo que foi apresentado até o momento e por resultados como os descritos por Eriksen, Björnstad e Götestam (1986) e Scheier, Botvin, Diaz e Griffin (1999), entende-se como mais admissível supor que algumas habilidades sociais específicas sejam favorecedoras, não necessariamente mantenedoras, da abstinência. Para dependentes químicos que buscam tratamento, possuir um bom repertório prévio e/ou incrementá-lo durante esse período poderia colaborar para a obtenção de melhores resultados. Isso não significa necessariamente ausência, obrigatória e duradoura, de consumo de álcool e outras drogas, mas um nível menor de envolvimento com tais substâncias.

Os dados apresentados neste artigo, ainda que expliquem apenas parte da relação entre habilidades sociais e envolvimento com substâncias psicoativas, são promissores por serem significativos e interpretáveis. Os resultados sugerem que intervenções em dependência química, planejadas para incluir o ensino de comportamentos socialmente habilidosos, permitem esperar maiores probabilidades de sucesso. Além de testar esta hipótese, pesquisas futuras devem buscar identificar com maior exatidão quais são esses comportamentos, quais estão intrinsecamente ligados ao envolvimento com álcool e quais com outras drogas.

Os estudos na área têm um longo caminho a percorrer. Diversas hipóteses precisam ser testadas, várias outras surgirão, mas o importante é que o resultado de tudo isso seja um melhor entendimento de como as relações interpessoais estão relacionadas ao envolvimento com substâncias psicoativas. E que isso leve ao aparecimento de novas alternativas de avaliação, prevenção e intervenção, permitindo que maior qualidade de vida possa ser alcançada por indivíduos que usam, abusam ou dependem de álcool ou qualquer outra droga, mas tenham o desejo de não fazê-lo mais.

\section{Questões metodológicas e limitações}

Os resultados apresentados neste artigo devem ser interpretados com reservas, considerando algumas questões importantes. A primeira delas refere-se ao uso de um instrumento de habilidades sociais gerais, não específicas ao contexto da dependência de álcool ou outras drogas. A escassez de métodos de medida das habilidades sociais e de enfrentamento, específicas para a dependência química, pode explicar a ausência de um maior número de resultados significativos absolutos e de resultados explicativos mais fortes neste estudo. Alguns autores (Litt, Kadden, Cooney, \& Kabela, 2003; Litt et al. 2008; Litt, Kadden, \& Kabella-Cormier, 2009) vêm relatando dificuldades em se medir o repertório de habilidades de enfrentamento para a dependência química com o uso de medidas mais globais. Embora comum, esta estratégia parece limitar o alcance dos resultados das pesquisas realizadas na área (Hasking \& Oei, 2007).

A recomendação, para uma possível solução do problema, seria a inclusão de comportamentos específicos de enfrentamento à dependência química (Forys et al., 2007). Del Prette e Del Prette (2009) alertam que a avaliação das habilidades sociais, dado seu caráter situacional-cultural, deve levar em conta os diferentes contextos, interlocutores, cultura e subcultura do indivíduo. Pode-se pensar, portanto, que o contexto da dependência química possui características bastante específicas, que não são captadas totalmente por instrumentos de medidas gerais de enfrentamento ou de habilidades sociais. Por isso, seria importante contar com um recurso de avaliação que seguisse as indicações da literatura da área, considerando situações específicas da dependência química.

A segunda limitação refere-se ao tipo de estudo e ao tamanho da amostra. Por se tratar de um estudo com característica essencialmente exploratória, o tamanho da amostra utilizada foi pequeno, o que não permite generalizações ou conclusões definitivas so- 
bre os resultados. Devem ser tratados como indícios de que existem importantes relações entre as variáveis que merecem ser investigadas mais a fundo e com mais cuidado.

A terceira refere-se à ausência de controle sobre o efeito de interações entre os diferentes tipos de drogas utilizadas pelos participantes da amostra. Na Dependência de Substâncias é pouco comum encontrar pessoas que consumam apenas um tipo de droga. Em geral, há o uso de pelo menos duas delas, ainda que legais, como a combinação álcool e nicotina. Como o instrumento foi usado ASSIST, que investiga o envolvimento com cada substância separadamente, os resultados não levaram em conta se a influência das habilidades sociais é diferente quando há o envolvimento com duas ou mais drogas simultaneamente.

\section{Agradecimentos}

Os autores agradecem o apoio da FAPESP- Fundação de Apoio à Pesquisa do Estado de São Paulo (Processo $n^{\circ}$ 2010/ 07890-3).

\section{REFERÊNCIAS}

American Psychiatric Association. (2002). Manual Diagnóstico e Estatístico de Transtornos Mentais - DSM-IV$R$. Porto Alegre: Artes Médicas.

Aliane, P. P., Lourenço, L. M., \& Ronzani, T. M. (2006). Estudo comparativo das habilidades sociais de dependentes e não dependentes de álcool. Psicologia em Estudo, 11, 83-88.

Araújo, R. B., Oliveira, M. S., Pedroso, R. S., \& Castro, M. G. T. (2009). Coping strategies for craving management in nicotine dependent patients. Revista Brasileira de Psiquiatria, 31(2), 89-94.

Ball, S. A., Todd, M., Tennen, H., Armelli, S., Mohr, C., Affleck, G., et al. (2007). Brief motivational enhancement and coping skills interventions for heavy drinking. Addictive Behaviors, 32, 1.105-1.118.

Barkin, S. L., Smith, K. S., \& DuRant, R. (2002). Social skills and attitudes associated with substance use behaviors among young adolescents. Journal of Adolescent Health, 30, 448-454.

Bordin, S., Zanelatto, N. A., Figlie, N. B., \& Laranjeira, R. (2010). Treinamento de habilidades sociais e de enfrentamento de situações de risco. In N.B. Figlie, S. Bordin \& R. Laranjeira (Orgs.), Aconselhamento em dependência química (pp. 313-353). São Paulo: Roca.

Budney, A. J., Roffman, R., Stephens, R. S., \& Walker, D. (2007). Marijuana dependence and its treatment. Addiction Science and Clinical Practice, December, 4-16.
Brown, S. A., Vik, P. W., McQuaid, J. R., Patterson, T. L., Irwin, M. R., \& Grant, I. (1990). Severity of psychosocial stress and outcome of alcoholism treatment. Journal of Abnormal Psychology, 99, 344-348.

Ciribelli, E. B., Luiz, A. M. A. G., Gorayeb, R., Domingos, N. A. M., \& Filho, A. B. M. (2008). Intervenção em sala de espera de ambulatório de dependência química: $\mathrm{Ca}$ racterização e avaliação de efeitos. Temas em Psicologia, 16(1), 107-118.

Cleveland, H. H., \& Harris, K. S. (2010). The role of coping in moderating within-day associations between negative triggers and substance use cravings: A daily diary investigation. Addictive Behaviors, 35, 60-63.

Cunha, S. M., Carvalho, J. N., Kolling, N. M., Silva, C. R., \& Kristensen, C. H. (2007). Habilidades sociais em alcoolistas: Um estudo exploratório. Revista Brasileira de Terapias Cognitivas, 3, 28-41.

Cunha, S. M., Peuker, A. C., \& Bizarro, L. (2012). Consumo de álcool de risco e repertório de habilidades sociais entre universitários. PSICO, 43(3), 289-297.

Del Prette, Z. A. P., \& Del Prette, A. (2001). Inventário de Habilidades Sociais (IHS-Del Prette): Manual de aplicação, apuração e interpretação. São Paulo: Casa do Psicólogo.

Del Prette, Z. A. P., \& Del Prette, A. (2005). Psicologia das habilidades sociais na infância: Teoria e prática. Petrópolis: Vozes.

Del Prette, Z. A. P., \& Del Prette, A. (2009). Avaliação de habilidades sociais: Bases conceituais, instrumentos e procedimentos. In Z. A. Del Prette \& A. Del Prette (Orgs.), Psicologia das habilidades sociais: Diversidade teórica e suas implicações (pp. 189-231). Petrópolis: Vozes.

Del Prette, Z. A. P., \& Del Prette, A. (2010). Habilidades Sociais e Análise do Comportamento: Proximidade histórica e atualidades. Perspectivas em Análise do Comportamento, 1(2), 104-115.

Donovan, D. M. (2009). Avaliação dos comportamentos dependentes na prevenção da recaída. In D.M. Donovan \& G.A. Marlatt (Orgs.), Avaliação dos comportamentos dependentes (pp. 1-50). São Paulo: Roca.

Eriksen, L. Björnstad, S., \& Götestam, K. G. (1986). Social skills training in groups for alcoholics: One-year treatment outcome for groups and individuals. Addictive Behaviors, 11, 309-329.

Felicíssimo, F. B., Casela, A. L. M., \& Ronzani, T. M. (2013). Habilidades sociais e alcoolismo: Uma revisão da literatura. Psicologia em Estudo, 18(1), 137-145.

Ferreira, M. P., \& Bernik, M. A. (2001). Dependência de substâncias. In B. Rangé (Org.), Psicoterapia comportamental e cognitiva de transtornos psiquiátricos (pp. 167-174). Campinas: Livro Pleno.

Forys, K., McKellar, J., \& Moos, R. (2007). Participation in specific treatment components predicts alcohol-specific 
and general coping skills. Addictive Behaviors, 32, 1.669-1.680.

Hasking, P. A., \& Oei, T. P. S. (2007). Alcohol expectancies, self-efficacy and coping in an alcohol-dependent sample. Addictive Behaviors, 32, 99-113.

Henrique, I. F., Micheli, D., Lacerda, R. B., Lacerda, L. A., \& Formigoni, M. L. (2004). Validação da versão brasileira do teste de triagem do envolvimento com álcool, cigarro e outras substâncias (ASSIST). Revista da Associação Médica Brasileira, 50, 199-206.

Kessler, F. H. P., Terra, M. B., Faller, S., Stolf, A. R., Peuker, A. C., Benzano, D., et al. (2012). Crack users show high rates of antisocial personality disorder, engagement in illegal activities and other psychosocial problems. The American Journal of Addictions, 21(4), 370-380.

Litt, M. D., Kadden, R. M., Cooney, N. L., \& Kabela, E. (2003). Coping skills and treatment outcomes in cognitive-behavioral and international group therapy for alcoholism. Journal of Consulting and Clinical Psychology, 71, 118-128.

Litt, M. D., Kadden, R. M., \& Kabela-Cormier, E. (2009). Individualized assessment and treatment program for alcohol dependence: Results of an initial study to train coping skills. Addiction, 104, 1.837-1.848.

Litt, M. D., Kadden, R. M., Kabela-Cormier, E., \& Petry, N. M. (2008). Coping skills training and contingency management treatments for marijuana dependence: Exploring mechanisms of behavior change. Addiction, 103(4), 638-648.

Mares, M. F., \& Torres, L. B. (2010). Mantenimiento de las habilidades de rehusarse al consumo en usuarios cronicos de alcohol y drogas: Um estúdio de casos. Salud Mental, 33(1), 47-55.

Marijuana Treatment Project Research Group (2004). Brief treatments for cannabis dependence: Findings from a randomized multisite trial. Journal of Consulting and Clinical Psychology, 72, 455-466.

Marlatt, G. A., \& Donovan, D. M. (2009). Prevenção da recaída: Estratégias de manutenção no tratamento de comportamentos adictivos. Porto Alegre: Artmed.

Marlatt, G. A., \& Witkiewitz, K. (2009). Problemas com álcool e drogas. In G.A. Marlatt \& D.M. Donovan (Orgs.), Prevenção da recaída: Estratégias de manutenção no tratamento de comportamentos adictivos (pp. 15-50). Porto Alegre: Artmed.

Mattoo, S. K., Chakrabarti, S., \& Anjaiah, M. (2009). Psychosocial factors associated with relapse in men with alcohol or opioid dependence. Indian Journal of Medical Research, 130, 702-708.

Monti, P. M., Abrams, D. B., Binkoff, J. A., Zwick, W. R., Liepman, M. R., Nirenberg, T. D. et al. (1990). Communication skills training, communication skills training with family and cognitive behavioral mood management training for alcoholics. Journal of Studies on Alcohol, 51, 263-270.

Monti, P. M., Kadden, R. M., Rohsenow, D. J., Cooney, N. L., \& Abrams, D. B. (2005). Tratando a dependência de álcool: Um guia de treinamento das habilidades de enfrentamento. São Paulo: Roca.

Monti, P. M., Rohsenow, D. J., Michalec, E., Martin, R. A., \& Abrams, D. B. (1997). Brief coping skills treatment for cocaine abuse: Substance use outcomes at three months. Addiction, 92(12), 1.717-1.728.

Murta, S. G., \& Tróccoli, B. T. (2005). Parando de beber: estágios de mudanças vividos por ex-alcoolistas. Estudos de Psicologia, 22(2), 157-166.

Sá, L. G. C., \& Del Prette, Z. A. P. (2012). Dependentes químicos sob tratamento ambulatorial: Análise de impacto sobre habilidades sociais. In M. B. Villa, A. A. R. Aguiar, \& Z. A. P. Del Prette (Orgs.). Intervenções baseadas em evidências: aplicações do Método JT (pp. 127-147). São Carlos: EDUFSCar.

Scheier, L. M., Botvin, G. J., Diaz, T., \& Griffin, K. W. (1999). Social skills, competence, and drug refusal efficacy as predictors of adolescent alcohol use. Journal of Drug Education, 29, 251-278.

Oei, T. P. S., \& Jackson, P. R. (1982). Social skills and cognitive behavioral approaches to the treatment of problem drinking. Journal of Studies on Alcohol, 43(5), 532-547.

Oliveira, P. A. (2010). Habilidades Sociais, Depressão, Ansiedade e Alcoolismo em Bombeiros: Um estudo correlacional. (Dissertação de Mestrado não-publicada) Universidade Federal de São Carlos, São Carlos.

Rangé, B., \& Marlatt, G. A. (2008). Terapia cognitivocomportamental de transtornos de abuso de álcool e drogas. Revista Brasileira de Psiquiatria, 30, 88-95.

Rohsenow, D. J., Martin, R. A., \& Monti, P. M. (2005). Urge-specific and lifestyle coping strategies of cocaine abusers: Relationships to treatment outcomes. Drug and Alcohol Dependence, 78, 211-219.

Sakiyama, H. M. T., Ribeiro, M., \& Padin, M. F. R. (2012). Prevenção de recaída e habilidades sociais. In M. Ribeiro \& R. Laranjeira (Orgs.), O tratamento do usuário de crack (pp. 337-350). Porto Alegre: Artmed.

Scheier, L. M., Botvin, G. J., Diaz, T., \& Griffin, K. W. (1999). Social skills, competence and drug refusal efficacy as predictors of adolescent alcohol use. Journal of Drug Education, 29(3), 251-278.

Van Hasselt, V. B., Hersen, M., \& Milliones, J. (1978). Social skills training in alcoholics and drug addicts: A review. Addictive Behaviors, 3, 221-233.

Villalba, E. A. (1995). Proyecto de entrenamiento en habilidades sociales con toxicómanos. In F.G. Rodríguez, J. M. L. Rubio \& L. J. Expósito (Orgs.), Habilidades sociales y salud (pp. 127-132). Madri: Biblioteca Eudema. 
Wagner, M. F., \& Oliveira, M. S. (2007). Habilidades sociais e abuso de drogas em adolescentes. Psicologia Clínica, 19(2), 101-116.

Wagner, M. F., \& Oliveira, M. S. (2009). Estudo das habilidades sociais em adolescentes usuários de maconha. Psicologia em Estudo, 14(1), 101-110.

Wagner, M. F., Silva, J. G., Zanettelo, L. B., \& Oliveira, M. S. (2010). O uso da maconha associado ao déficit de habilidades sociais em adolescentes. Revista Eletrônica Saúde Mental, Álcool e Drogas, 6(2), 255-273.
Witkiewitz, K., Villarroel, N. A., Hartzler, B., \& Donovan, D. M. (2011). Drinking outcomes following drink refusal skills training: Differential effects for African American and non-hispanic white clients. Psychology of Addictive Behaviors, 25(1), 162-167.

\section{Nota:}

1 O conjunto desses estudos pode ser encontrado em:

<http://www.rihs.ufscar.br/avaliacao-de-hs/ihs-del-prette/citacoes-do-ihs-del-prette>. 